\title{
Liposome-Glass Interaction. Influence of the Lipid Bilayer Phase State
}

\author{
B.G. TENCHOV ${ }^{1}$, D.N. PETSEV ${ }^{2}$, R.D. KOYNOVA ${ }^{1}$, C.S. VASSILIEFF ${ }^{2}$, H.W. MEYER ${ }^{3}$ \\ and J. WUNDERLICH ${ }^{3}$ \\ ${ }^{1}$ Central Laboratory of Biophysics, Bulgarian Academy of Sciences, 1113 Sofia (Bulgaria) \\ ${ }^{2}$ Faculty of Chemistry, University of Sofia, 1126 Sofia (Bulgaria) \\ ${ }^{3}$ Friedrich-Schiller-Universität, 6900 Jena (G.D.R.)
}

(Received 2 November 1988; accepted 7 February 1989)

\begin{abstract}
The adsorption of dipalmitoylphosphatidylcholine (DPPC) and palmitic acid from multilamellar lipid dispersions in excess water onto hydrophilic and hydrophobic glass surfaces was studied. Adsorption isotherms at different temperatures were obtained. The adsorbed lipid amount at saturation, $\Gamma_{\infty}$, was found to depend strongly on the phase state of the lipid dispersion. For DPPC, a sharp decrease of $\Gamma_{\infty}$ was observed at the gel-liquid crystal phase transition at $41^{\circ} \mathrm{C}\left(\mathrm{P}_{\beta^{\prime}}-\mathrm{L}_{\alpha}\right)$. The DPPC adsorption from the gel phases $\mathrm{L}_{\beta^{\prime}}$ and $\mathrm{P}_{\beta^{\prime}}$ was significantly greater than that from the low-temperature crystalline $\mathrm{L}_{c}$ phase. The $\mathrm{L}_{\mathrm{c}}$-isotropic phase transition of palmitic acid was also clearly reflected in the temperature dependence of $\Gamma_{\infty}$ for this lipid. Electron microscopy yielded direct evidence for the presence of lipid aggregates and restructured liposomes on the glass surface. In combination with the adsorption data, this shows that fluid liposomes bind less to glass surfaces than solid liposomes.
\end{abstract}

\section{INTRODUCTION}

Recently, Jackson et al. [1-3] have studied phospholipid adsorption onto solid surfaces. They have pointed out existing controversy concerning the mode of adsorption from liposomal suspensions: in contrast to well-defined layered structures obtained by means of the Langmuir-Blodgett technique [4-6], one may expect a complicated structure of the adsorbed lipid when a solid surface is brought into contact with an aqueous liposomal suspension. Here we present experimental results for dipalmitoylphosphatidylcholine (DPPC) and palmitic acid adsorption from aqueous suspensions of multilamellar lipid aggregates onto hydrophilic and hydrophobic flat glass surfaces.

Electron microscopy yields direct evidence for the presence of lipid aggregates on the surface. Further we follow in more detail the temperature dependence of multilamellar lipid vesicles adsorption, revealing a sharp change in the adsorption corresponding to the gel-liquid crystal phase transition in the 
lipid bilayer ( similar to the one reported in Ref. [3] for small unilamellar vesicles of DPPC). This fact may be of great biological interest since lipid bilayer fluidization has been shown to reduce cell-lipid bilayer adhesiveness [7-13].

\section{EXPERIMENTAL}

\section{Glass treatment}

Microscope cover glasses (Hirschmann, F.R.G.) were used. Hydrophilic and hydrophobic glass surfaces were prepared according to procedures described previously $[14,15]$. Hydrophilization was achieved by a 2 min treatment with a solution of $4 \mathrm{vol} . \%$ hydrofluoric acid and $50 \mathrm{vol} . \%$ nitric acid in water followed by extensive rinsing with doubly distilled water. Hydrophobization involved boiling the slides for $2 \mathrm{~h}$ in 5 vol.\% solution of dimethyldichlorsilane (DMDS) in dry toluene. After this the slides were washed successively with pure toluene, methanol and doubly distilled water. Finally, the slides were dried at $150^{\circ} \mathrm{C}$.

\section{Liposome suspensions}

DPPC (Fluka) and palmitic acid (Fluka, GC standard) were mixed with 0.1 mol.\% of the respective ${ }^{14} \mathrm{C}$-labelled compound (Amersham, U.K.). The lipids were hydrated overnight in an excess of doubly distilled water (pH 5.5, conductivity $\left.10^{-6} \Omega^{-1} \mathrm{~cm}^{-1}\right)$, then equilibrated for $1 \mathrm{~h}$ at $10^{\circ} \mathrm{C}$ above the respective melting phase transition temperatures $\left(41^{\circ} \mathrm{C}\right.$ for DPPC and $61^{\circ} \mathrm{C}$ for palmitic acid) and shaken at this temperature on a vortex mixer five times for 1 min with 2 min intervals. In the low-temperature measurements the DPPC suspension was pre-equilibrated for 4 days at $4{ }^{\circ} \mathrm{C}$ to allow for the formation of the subgel lamellar crystalline phase $\left(\mathrm{L}_{\mathrm{c}}\right)$.

\section{Lipid adsorption}

The glass slides were placed vertically in glass vessels containing lipid dispersions, pre-equilibrated at various temperatures. The lipid amount in the aqueous phase was always in great excess with respect to the adsorbed amount. This ensured constant bulk concentration during the adsorption process ( the concentration decrease was less than $0.1 \%$ ). After termination of adsorption the slides were rinsed with doubly distilled water.

The adsorbed amount of ${ }^{14} \mathrm{C}$-labelled lipid was determined using a LKB1215 scintilation counter. For this purpose the slides were transferred into vials containing toluene based scintilation liquid. The vials were vortexed for better dissolution of the lipid. It was assumed that labelled and unlabelled lipids have identical adsorptive properties.

The bulk lipid concentration and the adsorption time were varied in order 
to determine the adsorption saturation with respect to these two parameters. The lipid adsorption saturates, in our experiments, after $1.5 \mathrm{~h}$ incubation of the glass slides, in good agreement with the value of $1 \mathrm{~h}$ reported by Jackson et al. [1]. Consequently, in all measurements reported hereafter, we used a constant incubation time of $2 \mathrm{~h}$.

\section{Electron microscopy}

After termination of adsorption as described above the rinsed slides were dried in air. Replicas from the surfaces were made in a Balzers device BAF $400 \mathrm{D}$ at room temperature by evaporation of platinum/carbon at an angle of $35^{\circ}$ and secondly of carbon at an angle of $90^{\circ}$. Using hot water $\left(95^{\circ} \mathrm{C}\right)$ or $40 \%$ hydrofluoric acid, the replicas could be separated from the glass and after cleaning with chloroform they were examined in a Tesla BS 500 electron microscope.

\section{Calorimetric measurements}

The thermotropic phase transitions in the lipid dispersions were recorded using a high-sensitivity DASM-1M microcalorimeter as described previously [16]. The heating rate was $0.5^{\circ} \mathrm{C} \mathrm{min}^{-1}$.

\section{RESULTS}

The adsorption isotherms of DPPC are presented in Fig. 1. Adsorption values are calculated dividing the bound lipid weight by the smooth surface area (determined from the macroscopic geometric dimensions of the slides).

Electron microscopy observation of the slides directly proves the presence of many (not isolated) liposomes, or similar lipid aggregates on both hydrophilic and hydrophobic glass surfaces. The distribution of lipid bodies on the

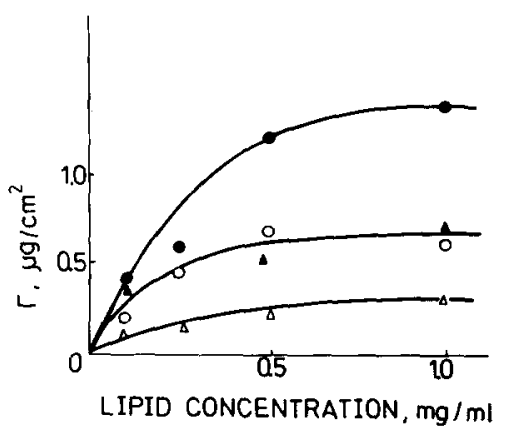

Fig. 1. Adsorption isotherms of DPPC: (๑) hydrophilic glass at $25^{\circ} \mathrm{C} ;(\mathrm{O})$ hydrophobic glass at $25^{\circ} \mathrm{C} ;(\Delta)$ hydrophilic glass at $50^{\circ} \mathrm{C} ;(\triangle)$ hydrophobic glass at $50^{\circ} \mathrm{C}$. 

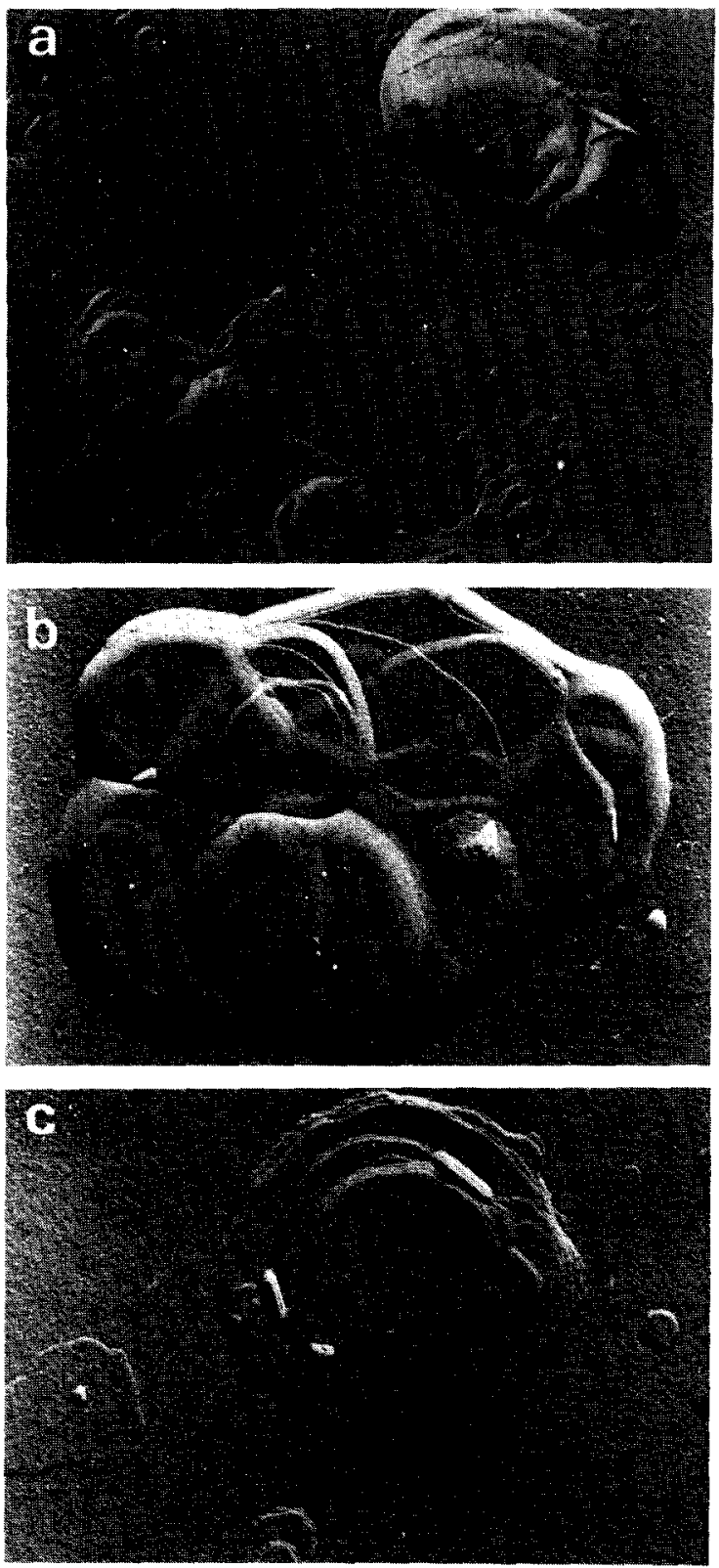

Fig. 2. Lipid bodies from DPPC suspension adsorbed onto hydrophilic glass surface at $50^{\circ} \mathrm{C}$, replicated after drying. There are well preserved liposomes ( $\mathrm{a}$ and $\mathrm{b}$ ) and more flattened material (a and $\mathrm{c}$ ). The bar represents $1 \mu \mathrm{m}$. 

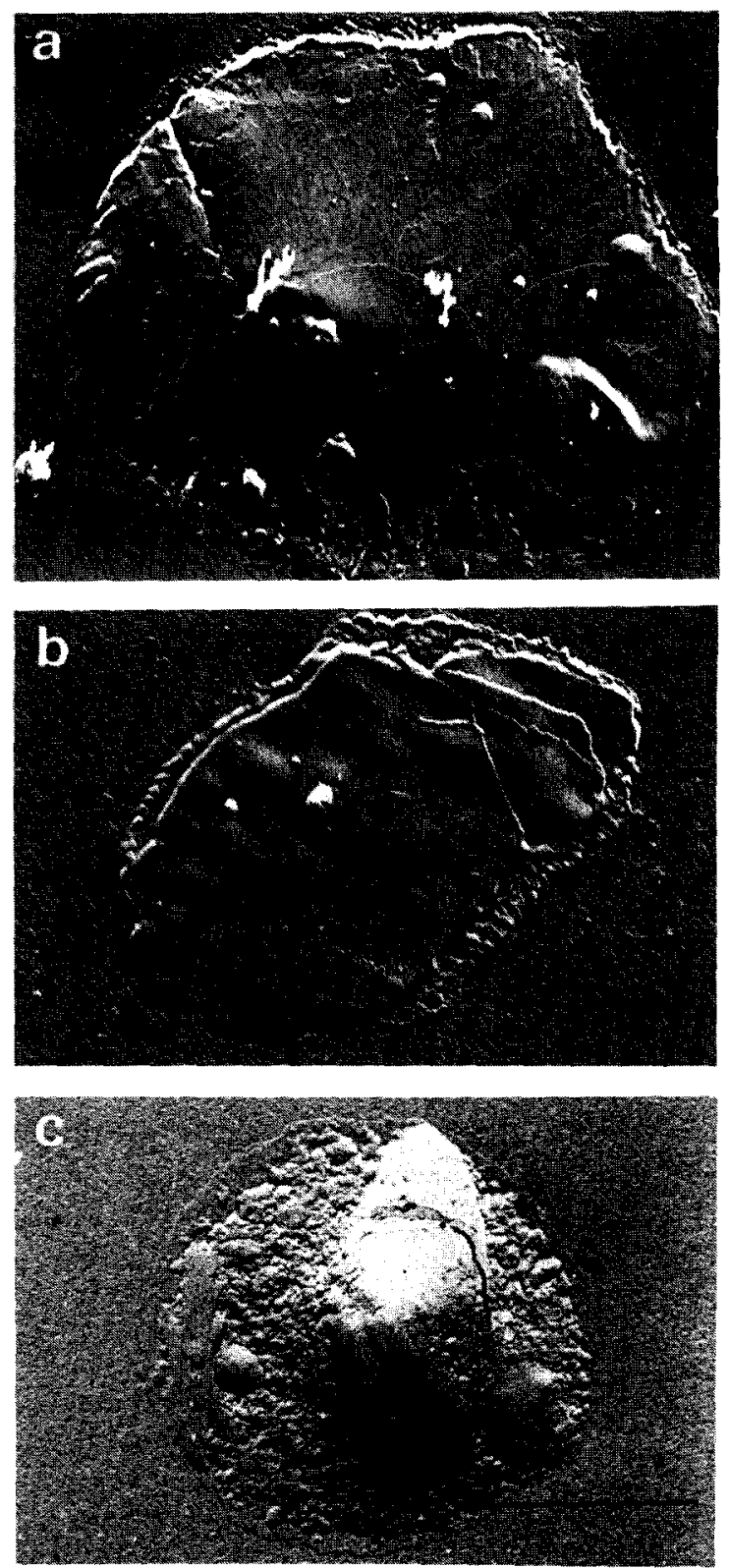

Fig. 3. Lipid bodies from palmitic acid suspension adsorbed onto hydrophobic glass surface at $70^{\circ} \mathrm{C}$, replicated after drying. The material has a layered ( $\mathrm{a}$ and $\mathrm{b}$ ) or a more granular (c) structure. The bar represents $1 \mu \mathrm{m}$. 
surfaces was not homogeneous enough to allow quantitative analysis of surface lipid density and differences with respect to glass pretreatment and temperature. There are differences in appearance of the dried lipid bodies on the glass surface in size and complexity of the structures (Figs 2 and 3). The alteration by drying includes the formation of flattened vesicles as well as layered structures of one or several layers thick.

The effect of temperature on the saturated lipid adsorption is shown in Fig. 4, upper panel, for DPPC, and in Fig. 5, upper panel, for palmitic acid (bulk concentration $1.35 \mathrm{mmol}^{-1}$ in both cases). The experimental points are the averages of two experimental series. The differences are of the order of the point size, except for the region of the phase transition where they are \pm 0.2 $\mu \mathrm{g} \mathrm{cm}{ }^{-2}$ for DPPC and $\pm 0.5 \mu \mathrm{g} \mathrm{cm}^{-2}$ for palmitic acid. Comparison with calorimetric data (Figs 4 and 5, lower panels) shows that sharp changes in
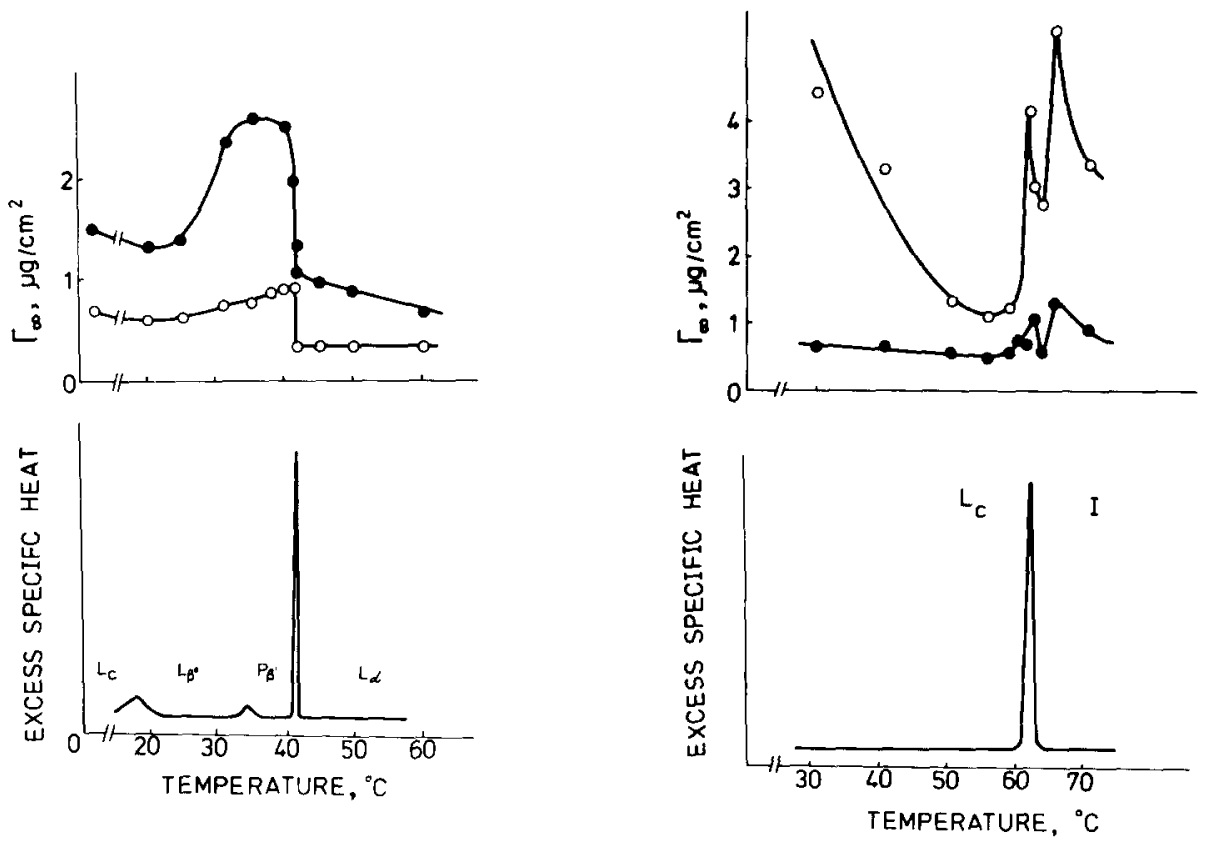

Fig. 4. (left) Upper panel: Temperature dependence of saturated DPPC adsorption; (๑) hydrophilic glass; $(O)$ hydrophobic glass. Lower panel: Phase transitions for DPPC recorded by differential scanning calorimetry; $\mathrm{L}_{c}$ subgel crystalline phase; $\mathrm{L}_{\beta}$ gel phase; $\mathrm{P}_{\beta}$ rippled gel phase; $\mathrm{L}_{\alpha}$ liquid crystalline phase.

Fig. 5. (right) Upper panel: Temperature dependence of saturated palmitic acid adsorption; ( ) hydrophilic glass; $(O)$ hydrophobic glass. Lower panel: Lamellar crystal $\left(\mathrm{L}_{c}\right)$-isotropic $(\mathrm{I})$ phase transition recorded by differential scanning calorimetry. 
adsorption occur during the melting phase transition (at $41^{\circ} \mathrm{C}$ for DPPC and $61^{\circ} \mathrm{C}$ for palmitic acid). In contrast to DPPC, palmitic acid adsorbs better onto hydrophobic glass (cf. Figs 4 and 5, upper panels).

\section{DISCUSSION}

Here adsorption values represent the amount of surface bound lipid per unit smooth area. Figures 2 and 3 are direct proof of the presence of lipid aggregates on the investigated surfaces. Hence it is meaningless to express our results in terms of layered adsorption as in Ref. [1].

Recent work [17-19] has shown large differences between the smooth and nitrogen (from BET measurements) area of solid surfaces for a large variety of nonporous solids, reflecting the surface roughness on a molecular scale. The latter can be quantified using the concept of surface fractal dimension [1719 ]. It rationalizes that on a molecularly rough surface, the surface area available for saturated adsorption depends on the size of adsorbing molecules. This complicates further the analysis (and comparison) of different lipid adsorption data. For instance, the monodisperse Ballotini beads (diameter $67 \pm 7 \mu \mathrm{m}$ ) used in Ref. [1] were found to have a krypton area of $0.109 \mathrm{~m}^{2} \mathrm{~g}^{-1}$ and a nitrogen area of $0.104 \mathrm{~m}^{2} \mathrm{~g}^{-1}$, whilst their smooth area is significantly smaller. Spherical beads with this diameter and a density* of $2.35 \mathrm{~g} \mathrm{~cm}^{-3}$ have a geometrical smooth area of $0.038 \mathrm{~m}^{2} \mathrm{~g}^{-1}$, which is about 3 times less than their nitrogen area. In the case of adsorption of lipid molecules or aggregates, the nitrogen and the smooth areas could be regarded, respectively, as upper and lower limits of the real available area.

\section{TABLE 1}

Results for saturated adsorption $\left(\Gamma_{\infty}\right)$ of DPPC liposomes

\begin{tabular}{lllll}
\hline & $\begin{array}{l}\text { Temperature } \\
\left({ }^{\circ} \mathrm{C}\right)\end{array}$ & \multicolumn{3}{c}{$\Gamma_{\infty}$, DPPC saturated adsorption $\left(\mu \mathrm{g} \mathrm{cm}^{-2}\right)$} \\
\cline { 3 - 5 } & & $\mathrm{a}$ & $\mathrm{b}$ & $\mathrm{c}$ \\
\hline Hydrophilic glass & 25 & 1.2 & 0.15 & 0.45 \\
Hydrophobic glass & 50 & 0.7 & 0.12 & 0.36 \\
& 25 & 0.7 & - & - \\
\hline
\end{tabular}

${ }^{a}$ On flat glass cover slides from multilamellar suspension; $\Gamma_{\infty}$ calculated with smooth area.

${ }^{b} \mathrm{On}$ Balotini beads from unilamellar suspension (data from Ref. [1]); $\Gamma_{\infty}$ calculated with krypton $\left(0.104 \mathrm{~m}^{2} \mathrm{~g}^{-1}\right)$ or nitrogen $\left(0.109 \mathrm{~m}^{2} \mathrm{~g}^{-1}\right)$ area.

cAs b but $\Gamma_{\infty}$ recalculated with smooth bead area $\left(0.038 \mathrm{~m}^{2} \mathrm{~g}^{-1}\right)$.

*The density of the actual Balotini sample is not quoted in Ref. [1]. The density of Balotini beads can vary in the range $2.0-2.8 \mathrm{~g} \mathrm{~cm}^{-3}$ depending on glass type and inclusion of gas bubbles in the beads [A. Nikolov, private communication]. 
Our results for saturated adsorption $\left(\Gamma_{\infty}\right)$ of DPPC liposomes are compared with those of Jackson et al. [1] in Table 1. A hypothetical dense monolayer (50 $\AA^{2}$ per DPPC molecule ) corresponds to $\Gamma_{\infty}=0.245 \mu \mathrm{g} \mathrm{cm}^{-2}$. Comparing this value with those in Table 1, illustrates the large indeterminacy in the layered adsorption concept. The expanded monolayer of column b (amount per nitrogen area) could be a dense monolayer in column c (amount per smooth area). If lipid aggregates are bound to the surface, both terms are misleading. Further speculations concerning differences between our $\Gamma_{\infty}$ values and those of Jackson et al. [1] (see Table 1) are not justified, due to the unknown properties of the glass surface and to other differences in the experimental conditions. We used multilamellar DPPC vesicles in doubly distilled water at $\mathrm{pH}$ 5.5 , whilst Jackson et al. used unilamellar vesicles in a medium with $\mathrm{pH} 7.43$ and an ionic strength of $0.0112 \mathrm{M}$. The electrolyte may suppress the electrostatic binding of the lipid to the surface.

Figures 4 and 5 depict the established correlation between multilamellar vesicle adsorption onto glass surfaces and the phase state of the lipid bilayer. A decrease of the lipid amount, bound to the glass surface during the melting transition, has also been observed for small unilamellar DPPC vesicles [3]. The sharp decrease in the DPPC amount bound to the surface at the gel-liquid crystal transition $\left(\mathrm{P}_{\beta^{\prime}}-\mathrm{L}_{\alpha}\right.$ fluidization, Fig. 4) correlates with some findings concerning cell adhesion: various types of cells adhere to solid lipid films but not to fluid films [20-22]; solid liposomes more strongly associated with cells than fluid liposomes [23,24]; fluidization of cell membranes reduces cell adhesiveness [7-13]. This, as well as the electron microscope investigation (Fig. 2 ), distinguishes liposome adhesion as the most important step in DPPC binding to solid surfaces. The conclusion that lipid adsorption via monomers is highly improbable was reached also in Ref. [2] following kinetic arguments. The formation of a monolayer (or bilayer) via vesicle disruption and spreading on the surface could not be proven for the multilamellar aggregates investigated here. Unilamellar liposomes do disrupt on adsorption [2]. Multilamellar ones are less likely to do so. On the other hand, the presence of vesicle fragments was proven even on a molecularly smooth liquid surface after the spreading of unilamellar sonicated liposomes [25]. The electron micrographs of the dried samples (Fig. 2) depict some spreading of the liposomes on the surface, which cannot be entirely ruled out in the wet state. In this respect it should be noted that for detergents the alternative between micellar and monomer adsorption onto solid surfaces is still unresolved [26].

As suggested recently $[27,28]$, a slight negative charge (about $4 \mathrm{mV}$ ) of the DPPC surface potential during the melting transition could be responsible for the decreased adhesiveness of the fluid liposomes. Another possible reason for this effect could be a steric repulsion predicted by Helfrich [29], due to outof-plane membrane fluctuations. This force should be suppressed in the solid state. It is appropriate, however, to note in this connection that adsorption 
from solid state DPPC is not constant with temperature but has a clearly expressed maximum in the rippled $\mathrm{P}_{\beta^{\prime}}$ phase (see Fig. 4).

The adsorption from palmitic acid suspension exhibits a complex temperature dependence, especially in the range of the lamellar crystal-isotropic transition which cannot be rationalized on the basis of the present measurements. Electron micrographs (Fig. 3 ) indicate that the adsorption process is dominated by aggregate adhesion (and possible spreading) rather than monomer adsorption.

We hope that further work will explain the exact physical cause of the observed phenomenon, yielding a sounder basis for understanding existing correlations between lipid phase state and cellular adhesiveness.

\section{ACKNOWLEDGEMENT}

We wish to thank the referee for the constructive criticism of the first version of the present work.

\section{REFERENCES}

1 S.M. Jackson, M.N. Jones and I.G. Lyle, Colloids Surfaces, 20 (1986) 171.

2 S.M. Jackson, M. Reboiras, I.G. Lyle and M.N. Jones, Faraday Discuss. Chem. Soc., 81 (1986) 291.

3 S.M. Jackson, M. Reboiras, I.G. Lyle and M.N. Jones, Colloids Surfaces, 27 (1987) 325.

4 K.B. Blodget and I. Langmuir, Phys. Rev., 51 (1937) 964.

5 O. Aldrecht, D.S. Johnston, C. Villaverde and D. Chapman, Biochim. Biophys. Acta, 687 (1982) 165.

6 L.K. Tamm and H.M. McConnell, Biophys. J., 47 (1985) 105.

7 J.C. Hoak, A.A. Spector, G.L. Fry and E.D. Warner, Nature, 228 (1970) 1330.

8 A.S.G. Curtis, C. Chandler and N. Picton, J. Cell. Sci., 18 (1975) 375.

9 B.E. Schaeffer and A.S.G. Curtis, J. Cell Sci., 26 (1977) 47.

10 E. Evans and A. Leung, J. Cell Biol., 98 (1984) 1201.

11 R.L. Hoover, R.D. Lynch and M.J. Karnovsky, Cell, 12 (1977) 295.

12 M.J. Karnovsky, A.M. Kleinfeld, R.L. Hoover, E.A. Dawidowicz, D.E. McIntyre, E.A. Salzman and R.D. Klausner, Ann. N.Y. Acad. Sci., 401 (1982) 61.

13 S. Kitagawa, J. Endo and F. Kametani, Biochim. Biophys. Acta, 798 (1984) 210.

14 H. Wolf and D. Gingel, Prog. Artif. Organs, 2 (1984) 844.

15 J. Tranchant (Ed.), Practical Manual of Gas Chromatography, 1969, Elsevier, Amsterdam, p. 100.

16 R.D. Koynova, A.I. Boyanov and B.G. Tenchov, Biochim. Biophys. Acta, 903 (1987) 186.

17 P. Pfeifer and D. Avnir, J. Chem. Phys., 79 (1983) 3558.

18 D. Avnir, D. Farin and P. Pfeifer, Nature, 308 (1984) 261.

19 P. Pfeifer, Appl. Surf. Sci., 18 (1984) 146.

20 O.Y Ivanova and L.B. Margolis, Nature, 242 (1973) 200.

21 L.B. Margolis, E.V. Dyatlovitskaya and L.D. Bergelson, Exp. Cell Res., 111 (1978) 454.

22 L.B. Margolis, A.N. Tikhonov and E.Y. Vasilieva, Cell, 19 (1980) 189.

23 R.E. Pagano and M. Takeichi, J. Cell Biol., 74 (1977) 531. 
24 F. Szoka, K. Jacobson, Z. Derzko and D. Paphadjopulos, Biochim. Biophys. Acta, 600 (1980) 1.

25 T. Ivanova, G. Georgiev, I. Panaiotov, M. Ivanova, M.A. Launois-Surpas, J.E. Proust and F. Puisieux, Prog. Colloid Polym. Sci., (1989), in press; M. Ivanova and I. Panaiotov, personal communication.

26 J.S. Clunie and B.T. Ingram, in G.D. Parfitt and C.H. Rochester (Eds), Adsorption from Solution at the Solid-Liquid Interface, Academic Press, New York, 1983, Chap. 3.

27 S.A. Tatulian, Biochim. Biophys. Acta, 736 (1983) 189.

28 S.A. Tatulian, Biochim. Biophys. Acta, 901 (1987) 161.

29 W. Helfrich, Z. Naturforsch., 33 (1978) 305. 